hypothesis that spinning neutrons will slow down, and no example of a pulsar speeding up its pulsation rate has been found. Some theoretical astronomers have felt all along that Gold's hypothesis is an oversimplification of what is happening in pulsars, and the new result might be a vindication of their doubts. Certainly it is not obvious what causes a spinning neutronstar to change its configuration so as to increase its rate of rotation. And in any case, there is doubt that the Gold mechanism of the emission of radiation from pulsars fully accounts for what is observed. In particular it is hard to understand how a hotspot can maintain its position on the star's surface for months on end to give the amazing regularity of the pulses. This is one aspect of the problem which the hypothesis of pulsations in a highly compressed star might better be able to explain. The production of radio pulses in strong gravitational fields such as must obtain on pulsars is only poorly understood, and has important implications for general relativity. The search will now be on for further eccentric behaviour in the other pulsars which are slowing down, chiefly the Crab Nebula pulsar which has the shortest period and the fastest slowdown rate of all.

\title{
New Antiviral Drug
}

EXPERIMENTs which promise a new approach to the chemotherapy of viral diseases are reported on page 273 of this issue by Heller and his colleagues. Their discovery, that the antibiotic rifampicin selectively inhibits the multiplication of vaccinia (pox) virus in cultured mouse cells, is bound to cause considerable excitement among animal virologists. This Israeli group seems to have won the race that has been developing between several groups investigating the possibilities of using rifampicin, a semi-synthetic derivative of the antibiotic rifamycin which is itself isolated from Streptomyces mediterraneus, as a selective antivira] agent. It seems certain that confirmatory reports will be rushed to the press in the next few months.

The first clue which suggested that rifampicin might be a clinically useful viricide came from work with bacteria which showed that the antibiotic kills bacterial cells by inhibiting the synthesis of RNA off DNA templates. In other words, the antibiotic stops the expression of genes. As experiments with bacterial cells and preparations of the enzyme responsible for RNA synthesis-DNA dependent RNA synthetase-have proved, it does this by interacting directly with the bacterial RNA synthetase. On the other hand, comparable experiments with mammalian cells and mammalian RNA synthetase showed that rifampicin neither inhibits mammalian RNA synthesis nor interacts with mammalian RNA synthetase. This, of course, immediately raised the possibility that the drug might prove to be a selective antiviral agent. All depended on whether or not the viral RNA synthetase more closely resembled its bacterial counterpart than its mammalian counterpart in its sensitivity to the antibiotic. Usually, chemicals which inhibit viral multiplication in mammalian cells also strongly inhibit the growth of the host cells and as a result are of no use in medicine, but it turns out that rifampicin will stop vaccinia virus from multiplying without killing the host cell.

Heller and his colleagues have yet to establish the precise mechanism of this inhibition, but it seems certain that it involves RNA synthesis. So far they have one experiment which supports but by no means confirms this notion. They find that RNA viruses art not inhibited by the drug. This is no surprise, because the RNA synthetase of RNA viruses, which copies RNA off an RNA instead of a DNA template, differ's from that of DNA viruses, including vaccinia virus. Apparently vaccinia virus is much less sensitive to rifampicin than are bacteria, and much larger doses of the antibiotic are needed to produce inhibition, but the crucial factor is that the viruses are far more sensitive to the drug than are mammalian cells.

\section{ATMOSPHERIC MEASUREMENTS}

\section{Background Pressure Fluctuations}

Some of the first results to have been obtained with the array of microbarographs operated by the Hudson Laboratories of Columbia University have now provided convincing evidence of a correlation between atmospheric pressure fluctuations and various meteorological phenomena, jet streams in particular. The array has already won attention for itself by the detection of pressure fluctuations from the launching of a Saturn rocket from Capo Kennedy last year (Science, 162, 1116 : 1968). It consists of some fifteen instruments strung out over several hundred kilometres covering most of New York State, with one station at Lebanon in the south and another on Long Island. The mierobarographs have a sensitivity of 3 microbars. Data are transmitted from the distant sites by means of a digital sampling device operating at intervals of between 1 and 10 seconds, and the instruments are adjusted so as to be most sensitive to fluctuations. The array is big enough not merely to distinguish between local and large-scale fluctuations, but also to determine the speed and direction of movement of substantial disturbances of the atmosphere. An account of the first observations by T. J. Herron. I. Tolstoy and D. W. Kraft appears in the Journal of Geophysicul Research (74, 1321; 1969). 